\title{
Radical Biocatalysis: Using Non-Natural Single Electron Transfer Mechanisms to Access New Enzymatic Functions
}

\author{
Todd K. Hyster* \\ Department of Chemistry, Princeton University, \\ Princeton, NJ 08544, USA \\ thyster@princeton.edu
}

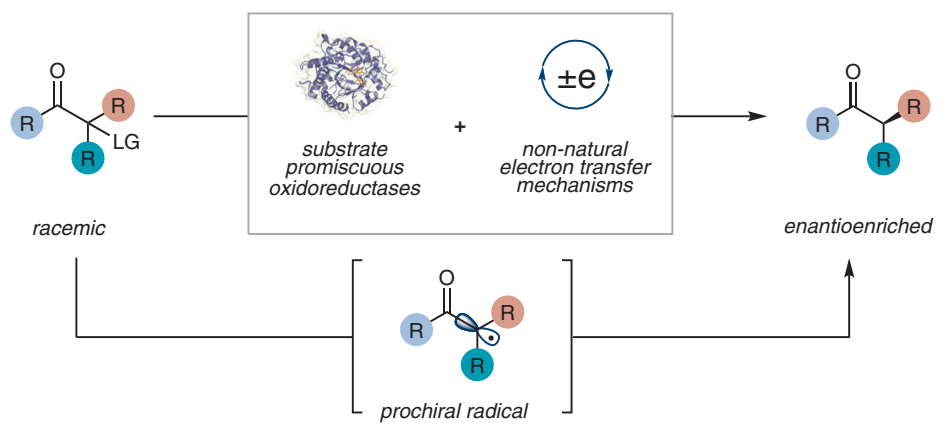

Received: 13.03 .2019

Accepted after revision: 14.04.2019

Published online: 07.05.2019

DOI: 10.1055/s-0037-1611818; Art ID: st-2019-p0146-sp

Abstract Exploiting non-natural reaction mechanisms within native enzymes is an emerging strategy for expanding the synthetic capabilities of biocatalysts. When coupled with modern protein engineering techniques, this approach holds great promise for biocatalysis to address long-standing selectivity and reactivity challenges in chemical synthesis. Controlling the stereochemical outcome of reactions involving radical intermediates, for instance, could benefit from biocatalytic solutions because these reactions are often difficult to control by using existing small molecule catalysts. General strategies for catalyzing nonnatural radical reactions within enzyme active sites are, however, undeveloped. In this account, we highlight three distinct strategies developed in our group that exploit non-natural single electron transfer mechanisms to unveil previously unknown radical biocatalytic functions. These strategies allow common oxidoreductases to be used to address the enduring synthetic challenge of asymmetric hydrogen atom transfer.

1 Introduction

2 Photoinduced Electron Transfer from NADPH

3 Ground State Electron Transfer from Flavin Hydroquinone

4 Enzymatic Redox Activation in NADPH-Dependent Oxidoreductases

5 Conclusion

Key words asymmetric catalysis, radical reactions, biocatalysis, photochemistry, single electron transfer, catalytic promiscuity

\section{Introduction}

Enzymes are attractive catalysts for asymmetric synthesis as they provide unmatched control over the stereochemical outcome of chemical reactions. As a consequence, enzymes are increasingly used in chemical synthesis, including in the preparation of pharmaceutically valuable molecules. ${ }^{1} \mathrm{~A}$ factor limiting the expanded use of enzymes in chemical synthesis is the narrow breadth of distinct chemical reactions catalyzed by enzymes. The primary

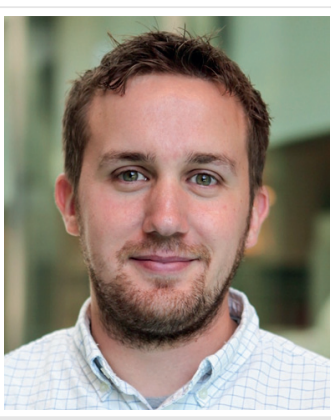

Todd Hyster is a native of Minnesota and obtained his BS in chemistry from the University of Minnesota - Twin Cities in 2008. He received his PhD from Colorado State University in 2013, working in the labs of Tomislav Rovis. He then went on to be a NIH postdoctoral fellow in the labs of Frances Arnold at Caltech. Since the fall of 2015, he has been an assistant professor in the chemistry department at Princeton University.

strategy for addressing this limitation is mining nature to discover new types of chemical reactivity. ${ }^{2}$ Although this approach has proven to be effective, it is intrinsically limited to reactivity patterns evolved in nature. To realize the full potential of enzymes to improve chemical synthesis, approaches need to be developed in which reactivity patterns currently unknown to nature may be catalyzed. ${ }^{3}$

Strategies for enabling enzymes to catalyze non-natural reactions have primarily relied on the catalytic promiscuity inherent to metalloenzymes and hydrolases. ${ }^{3 c, 4}$ In the case of metalloenzymes, organometallic cofactors located within enzyme active sites are responsible for providing the desired reactivity while the protein scaffold provides enantioand diastereoselectivity (Figure 1). ${ }^{5}$ Alternatively, the catalytic promiscuity of hydrolases relies on the ability of the oxyanion hole to catalyze other reactions that proceed via oxyanionic intermediates (Figure 1 ). ${ }^{6}$ Inspired by these examples, we sought to develop strategies to enable enzymes 
to catalyze new reactions mediated by non-natural freeradical intermediates. These represented a family of reactivity for which robust enzymatic catalysts had not been developed. Moreover, as controlling the stereochemical outcome of radical reactions remains a challenge for small molecule catalysts, we anticipated that a biocatalytic solution would be of high synthetic value (Figure 1 ). ${ }^{7}$

Central to our goal is the development of general strategies for forming radical species in enzyme active sites. We ruled out intercepting radical intermediates formed by monooxygenases, such as P450s, because of their exceedingly short lifetimes. ${ }^{8}$ Alternatively, the radical SAM family of enzymes, while capable of generating synthetically versatile radical intermediates, are typically plagued by poor substrate scope and sensitivity to oxygen, thus limiting their application in chemical synthesis. ${ }^{9}$ Instead, we sought to develop a biocatalytic method that would adopt the characteristics of the most ubiquitous enzymes in chemical synthesis: namely stability and substrate promiscuity. We identified commonly used oxidoreductases as attractive scaffolds for their broad use in organic synthesis (Figure 1). If a non-natural electron transfer event could be induced to occur within their active sites, it would enable existing collections of these enzymes to be quickly applied to non-natural reactions. This approach would also significantly lower the barrier for reaction adoption because libraries of oxidoreductases can be purchased from many chemical vendors.

\section{Photoinduced Electron Transfer from NADPH}

Nicotinamide adenine dinucleotide (NADH) is a biological reductant that functions as a hydride donor for many synthetically valuable oxidoreductases. ${ }^{10}$ In an effort to understand its biological function, a variety of NADH analogues have been prepared, including $N$-benzyl nicotinamide (BNAH) and Hantzsch esters. ${ }^{11}$ While these analogues typically function as hydride donors in chemical synthesis, Fukuzumi found that when irradiated with visible light, BNAH was promoted to is excited triplet state in which it would function as a potent single electron reduc$\operatorname{tant}\left(E_{0 x}{ }^{*}=-2.60 \mathrm{~V}\right) .{ }^{12}$ This function was demonstrated in a series of dehalogenation reactions in which $\mathrm{BNAH}^{*}$ can reductively cleave $\mathrm{C}-\mathrm{Br}$ bonds to afford alkyl radicals that readily abstract a hydrogen atom from BNAH to afford the reduced product (Scheme 1). We recognized that if NADH displayed a similar reactivity profile, it would be possible to render radical dehalogenations asymmetric using nicotin-

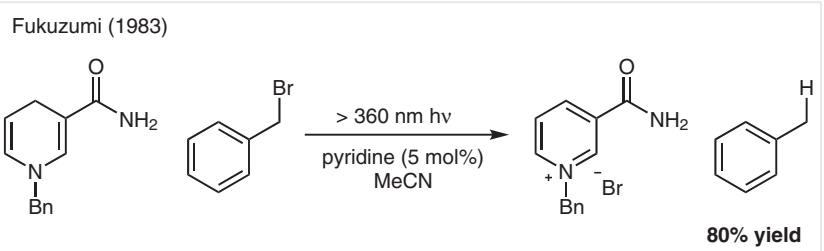

Scheme 1 Fukuzumi's photodehalogenation of benzyl bromide with $\mathrm{BNAH}$

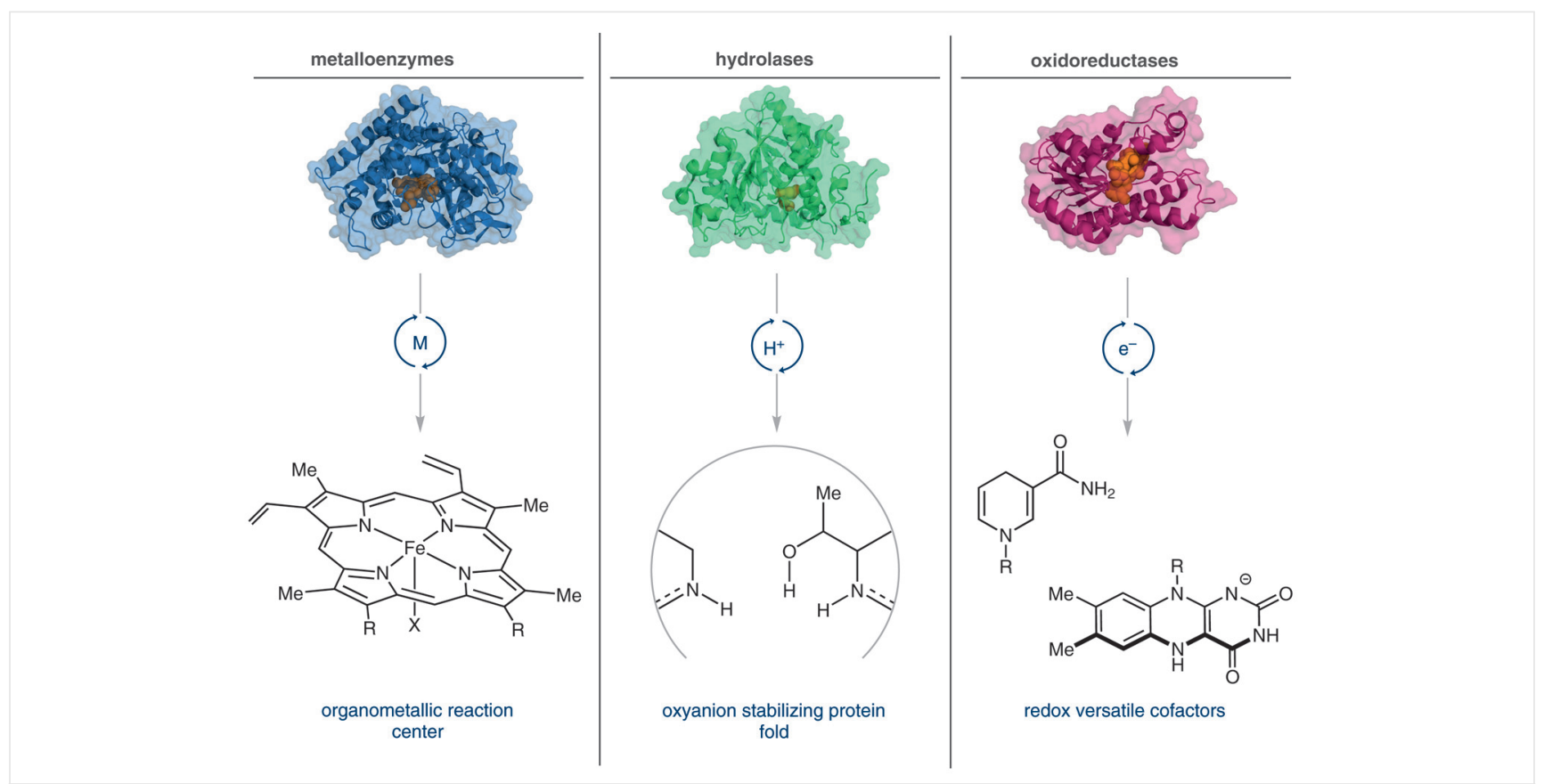

Figure 1 Strategies for revealing non-natural enzyme functions 
amide-dependent ketoreductases (KREDs). In this scenario, the protein scaffold would be responsible for controlling the delivery of a hydrogen atom to an intermediary prochiral radical. Asymmetric delivery of hydrogen atom represents a long-standing challenge in chemical synthesis for which small molecule reagents have proven largely ineffective at delivering products with synthetically useful levels of enantioselectivity. ${ }^{13}$

We began by testing a collection of engineered KREDs from Codexis. ${ }^{14}$ As a model reaction we explored the dehalogenation of $\alpha$-bromo- $\alpha$-aryl lactones because of their modest reduction potentials and the presence of a carbonyl functional group permitting substrate binding. By using standard reaction conditions for KREDs and irradiating with blue light, the desired dehalogenation product was observed, with Codexis P2D03 providing product in $81 \%$ yield with 98:2 er favoring the $R$ enantiomer (Scheme 2). Structural information, generously provided by Gjalt Huisman at Codexis, revealed the most active KREDs to be variants of the short-chain dehydrogenase from $L$. kefir which possesses an expanded active site. ${ }^{15}$ With this knowledge in hand, we engineered a KRED from L. kefir (LKADH-E145F-F147LY190F) capable of catalyzing the reaction with high levels of enantioselectivity (Scheme 2). We hypothesized that naturally occurring KREDs with large active sites should also be capable of catalyzing this dehalogenation reaction. Indeed, when a 'bulky-bulky' short-chain dehydrogenase from Ralstonia (RasADH) was subjected to the reaction conditions, the desired dehalogenated product was formed with good levels of enantioselectivity, but instead favoring the $S$ enantiomer (Scheme 2). ${ }^{16}$

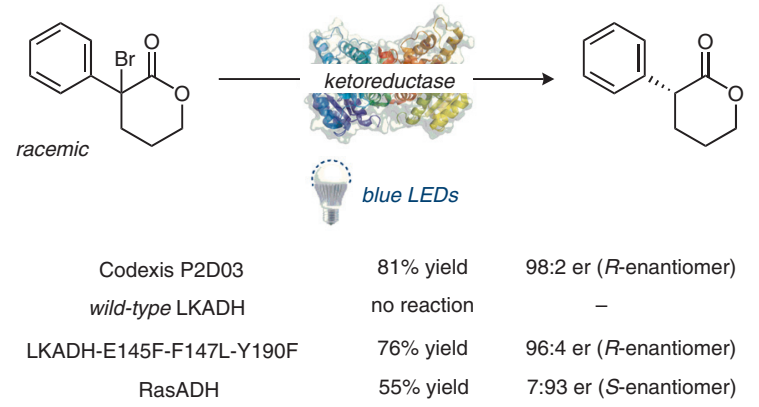

Scheme 2 Results for KRED-catalyzed asymmetric radical dehalogenation

In our initial optimization, we identified blue light to be ideal for the dehalogenation reactions. This came as a surprise as the $\lambda_{\max }$ for NADH is $340 \mathrm{~nm}$. To better understand the fundamentals of the photoinduced electron transfer event, we conducted a series of UV/Vis experiments. An initial evaluation of the emission spectra of the LEDs with the absorption spectra of the substrate and NADPH/KRED complex confirmed that neither direct excitation of the substrate nor the secondary complex is responsible for electron transfer (Scheme 3). When the NADPH/KRED complex spectrum was subtracted from the NADPH/KRED/substrate spectrum, a new broad absorption feature at $395 \mathrm{~nm}$ was observed. We attributed this new absorption feature to the formation of an electron donor-acceptor (EDA) complex formed between NADPH and the substrate within the enzyme active site. Control experiments confirm that this complex does not form in the absence of enzyme, providing a mechanism for gating electron transfer to occur only in the enzyme. Although this mechanism for electron transfer has experienced renewed interest in the preceding decade, it has never before been observed for biocatalytic reactions. $^{17}$

A survey of the substrate scope of the Codexis KRED P2D03 established the generality of this approach (Scheme $4)$. A variety of substituted $\alpha$-aryl- $\delta$-valerolactones can be effectively dehalogenated under the standard reaction conditions. Moreover, $\gamma$-butyrolactones can also be dehalogenated, albeit with slightly diminished enantioselectivities. Finally, substrates with $\alpha$-benzylic substituents can also be dehalogenated in modest yield but good levels of enantioselectivity. The short chain dehydrogenase from Cupriavidus $s p$. (RasADH) is capable of reducing a similar array of substrates, albeit with diminished yields. These enantioenriched lactones are challenging to prepare with use of traditional synthetic methods, such as asymmetric $\alpha$-arylation
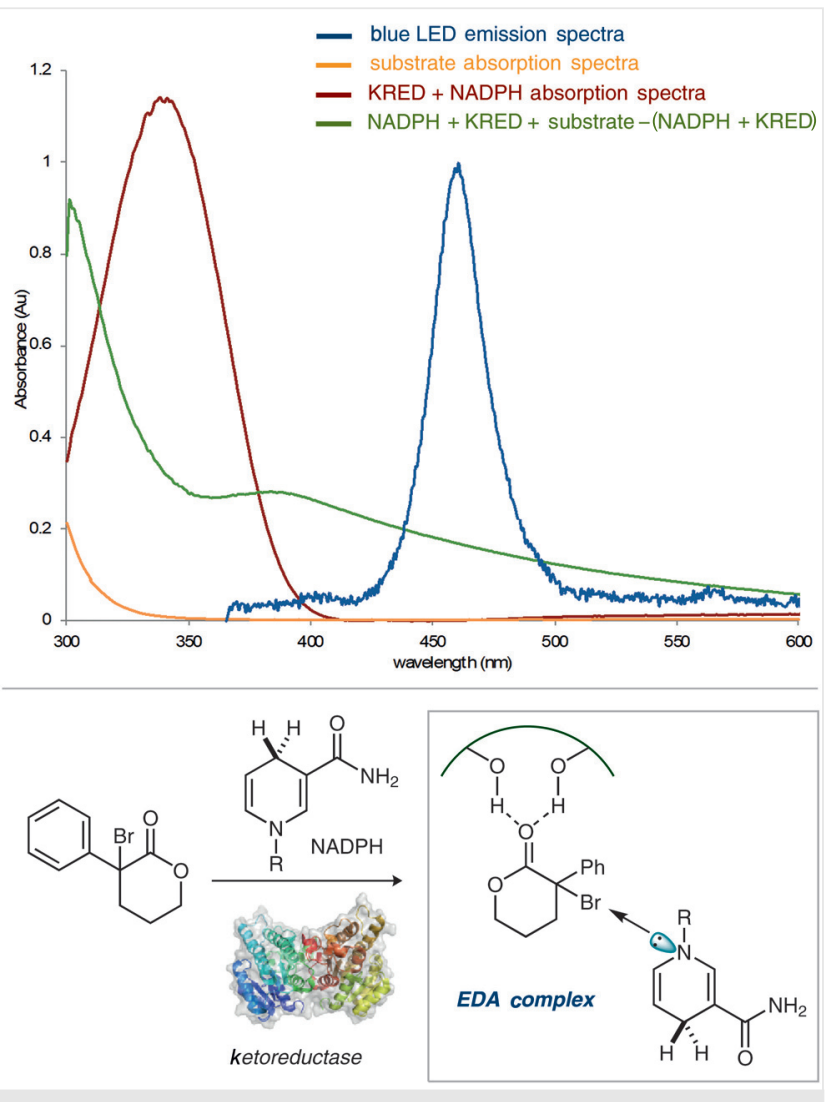

Scheme 3 Mechanistic studies 
reactions. ${ }^{18}$ We imagine that a two-step halogenation/dehalogenation deracemization protocol may be an attractive strategy for the preparation of enantioenriched products.

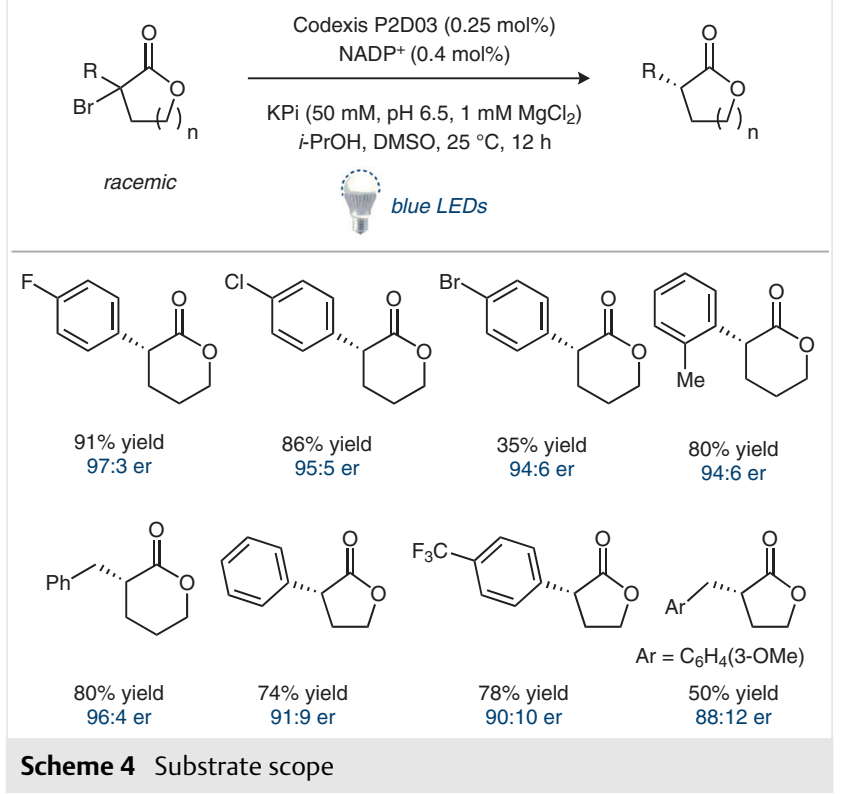

\section{Ground State Electron Transfer from Fla- vin Hydroquinone}

Next, we shifted to determining whether flavin-dependent 'ene'-reductases (EREDs) could catalyze radical reactions. These enzymes are responsible for catalyzing the stereoselective reduction of enones and other activated alkenes. ${ }^{19}$ Mechanistically, this reaction occurs through hydride transfer from the flavin hydroquinone $\left(\mathrm{FMN}_{\mathrm{hq}}\right)$ to the electrophilic position of the enone, followed by selective protonation of the resulting enolate to afford product. While this reactivity proceeds exclusively through the quinone and hydroquinone oxidation states of flavin, flavoproteins responsible for cellular respiration are able to exploit the open-shell semiquinone oxidation state to mediate single electron transfers. ${ }^{20}$ We recognized that if the flavin cofactor in EREDs could use the flavin semiquinone to mediate single electron transfer, it would be possible to use EREDs as catalysts for radical reactivity. In this scenario, we imagined that $\mathrm{FMN}_{\mathrm{hq}}$ could serve as a single electron reductant. We anticipated EREDs would not require photoexcitation to drive the single electron reduction because of the low oxidation potential of $\mathrm{FMN}_{\mathrm{hq}}\left(E_{\mathrm{ox}}=-450 \mathrm{mV}\right)$ by comparison to that of $\mathrm{NADPH}\left(E_{\mathrm{ox}}=570 \mathrm{mV}\right)$.

As a test for the proposed reactivity, we sought to dehalogenate $\alpha$-bromophenylacetic ester derivatives. ${ }^{21}$ These substrates were selected because they provided poor levels of enantioselectivity when dehalogenated by KREDs. Mechanistically, we anticipated $\mathrm{FMN}_{\mathrm{hq}}$ could directly reduce the substrate upon binding to the ERED active site. After meso- lytic cleavage, the resulting prochiral radical can abstract a hydrogen atom from the weak $\mathrm{N} 5-\mathrm{H}$ bond of the flavin semiquinone to afford the dehalogenated product (Scheme 5 ). In an initial screen of enzymes, we found that the ERED from Gluconobacter oxydans (GluER) provided the highest levels of enantioselectivity, however, with modest yield (69\% yield, 88:12 er). Recognizing that these enzymes could be valuable in setting these challenging stereocenters, we conducted site saturation mutagenesis on positions lining the enzyme active site. We quickly found that mutations to the conserved tyrosine furnished the most significant improvements, with Y177F affording the product in $89 \%$ yield with 98:2 er.

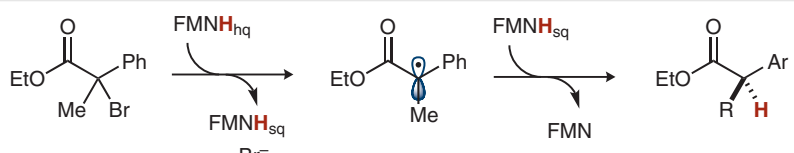

$$
\begin{aligned}
& \text { electron transfer/ hydrogen atom } \\
& \text { mesolytic cleavage transfer } \\
& \text { (m) }
\end{aligned}
$$

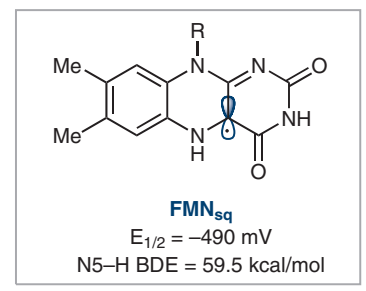

Scheme 5 ERED-catalyzed radical dehalogenation

The scope of this transformation proved to be quite broad, with a variety of meta- and ortho-substituted arenes providing product in good yield and enantioselectivity (Scheme 6). Furthermore, the system has proven to be quite broad with regards to substitution at the $\alpha$ position with various alkyl groups being tolerated. Interestingly, a fluorine substituent is tolerated at the $\alpha$ position without undergoing dehalogenation, allowing for the synthesis of enantioenriched $\alpha$-fluoro esters. Finally, heterocycles proved to be effective substrates for this reaction, although they produced product with suboptimal levels of enantioselectivity.

Studies were conducted to help elucidate the mechanism of dehalogenation (Scheme 7). Isotopic labeling experiments were conducted to determine the terminal source of hydrogen atom. When flavin was labeled, we observed $85 \%$ deuterium incorporation in the product, suggesting that flavin serves as the source of hydrogen atom in our best variant. Interestingly, when the same study was conducted with a poorly enantioselective enzyme, old yellow enzyme 1 (OYE1), no deuterium incorporation was observed. In these poorly selective variants, we hypothesize that the semiquinone functions as a single electron reductant rather than hydrogen atom source. In this scenario, the stereode- 


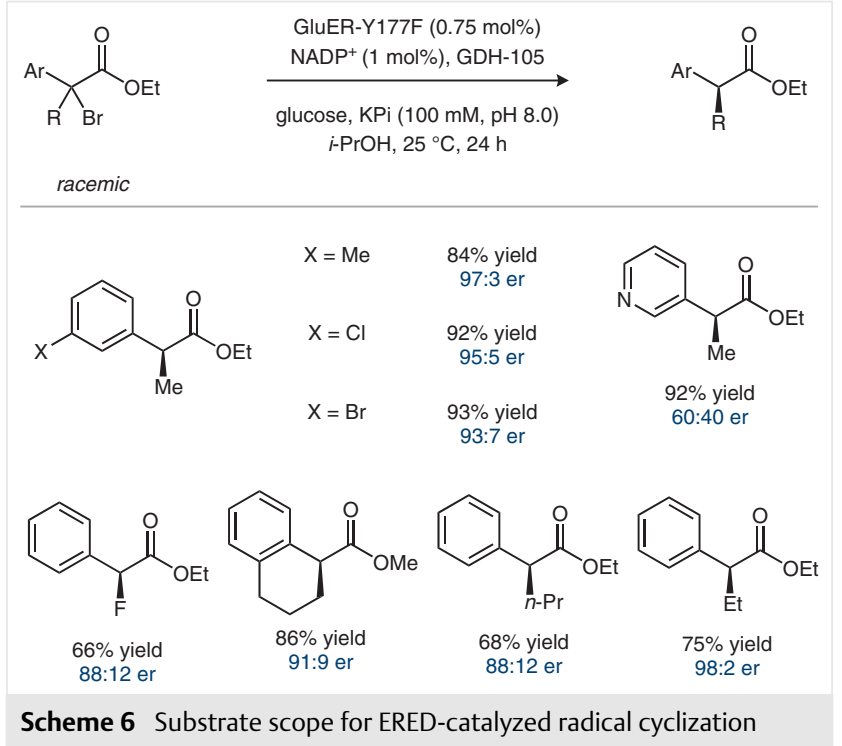

termining step is protonation of an enolate. We hypothesize that the presence of multiple proton sources within the enzyme active site (protic side chains and solvent) potentially account for the poor levels of enantioselectivity observed under this mechanism.

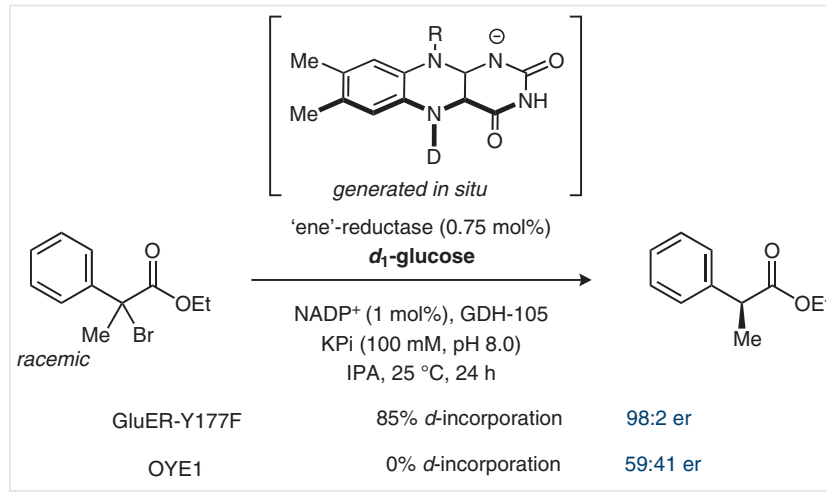

Scheme 7 Isotope incorporation experiments

\section{Enzymatic Redox Activation}

We recognized that a limitation to our previous strategies was a lack of tunability with regards to the redox capabilities of biological cofactors. In the case of the nicotinamide-dependent chemistry, electron transfer required the formation of an EDA complex, which can be sensitive to changes in the electronic properties of the cofactor. The flavin hydroquinone, while not requiring an EDA complex, was limited to electronically activated substrates. We hypothesized that a more modular approach would be to use an exogenous reductant to facilitate electron transfer and a general protein scaffold as a chiral catalyst. The challenge inherent to this approach is the development of gating strategies to ensure that radical formation only occurs within the enzyme active site. We were inspired by the role Lewis and Brønsted acids play in attenuating the reduction potentials of substrates with Lewis basic functional groups in organic synthesis. ${ }^{22}$ We hypothesized that the hydrogen bonds involved in substrate binding could have a similar effect in enzyme catalysts. If so, it would be possible to use substrate/photoredox catalyst combinations where electron transfer in solution would be challenging. However, upon binding to the enzyme active site, the substrate would be electronically activated for reduction (Scheme 8).

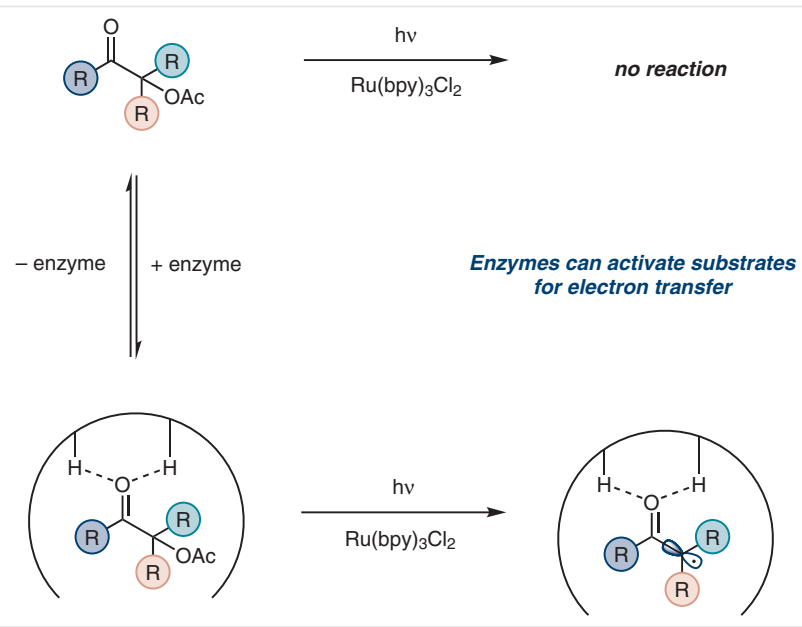

Scheme 8 Enzymatic redox activation

We tested this hypothesis of enzymatic redox activation on a reductive radical deacetoxylation reaction. Falvey previously reported that strong photoreductants are able to deacetoxylate $\alpha$-acetoxyl ketones. ${ }^{23}$ Mechanistically this occurs through reduction of the ketone to a ketyl radical followed by loss of acetate through a spin-center shift to form an $\alpha$-acyl radical that is quenched by reduction to the enolate followed by protonation. It has been observed, however, that in the presence of hydrogen bond donors, the redox potential of the substrate can be significantly attenuated. We hypothesized that the active site of an enzyme should play a similar role in activating a substrate for electron transfer.

Initial studies were conducted on the deacetoxylation of tetralone derivatives. We selected a nicotinamide-dependent double bond reductase (DBR) as an enzymatic scaffold for this reactivity because this enzyme does not reduce carbonyls and is reported to be substrate promiscuous. ${ }^{24}$ Control experiments revealed that no reaction was observed in the absence of a photocatalyst (Scheme 9). However, upon addition of commonly used transition-metal and organic photocatalysts, deacetoxylation was observed with good levels of enantioselectivity. While the water-soluble $\mathrm{Ru}(\mathrm{bpy})_{3} \mathrm{Cl}_{2}$ provided the best levels of enantioselectivity, 
<smiles>CC1(O)CCc2ccccc2C1=O</smiles>

racemic$$
\text { racenic }
$$

no photocatalys

$\mathrm{Ru}(\mathrm{bpy})_{3} \mathrm{Cl}_{2}$

Eosin $Y$

Rose Bengal
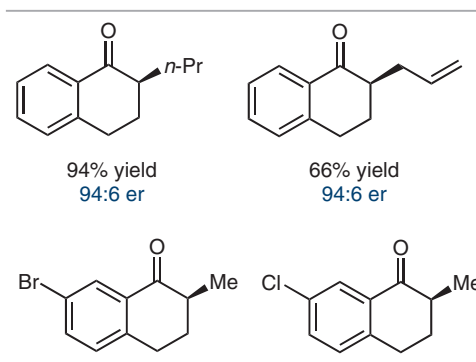

$63 \%$ yield
$97: 3 \mathrm{er}$

Me

$69 \%$ yield 81:19 er

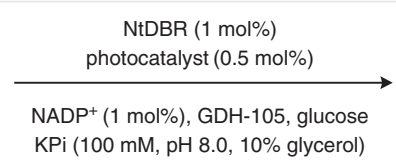

green LEDs<smiles>CC1CCc2ccccc2C1=O</smiles>

$-$

$96: 4$ er

$93: 7$ er

93:7 er

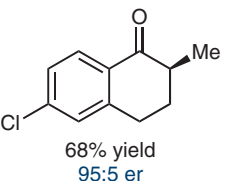

$15 \%$ yield

$86 \%$ yield<smiles>O=C1c2ccccc2CC[C@@H]1Br</smiles>

95:5 er<smiles>C[C@H]1CCCc2ccccc2C1=O</smiles><smiles>CCC(C)C(=O)c1cccc(OC)c1</smiles>

$44 \%$ yield

$38 \%$ yield 88:12 er

Scheme 9 ERA substrate scope and photocatalyst screen

we ultimately found Rose Bengal to be the most effective, providing product in good yields and with good levels of enantioselectivity. A survey of the substrate scope revealed this activation mode to be effective for a variety of tetralone derivatives. Furthermore, acetophenone derivatives could also be dehalogenated, although product was formed in much lower yields.

A series of mechanistic studies were performed to better understand the role the enzyme plays in activating the substrate for electron transfer. As all intermediates after the spin-center-shift are prochiral, we hypothesized that if binding was required for electron transfer a kinetic resolution of the starting material should be observed. Indeed, when the unreacted starting material was isolated we found that it was enriched in one enantiomer, confirming the importance of enzyme binding for electron transfer to occur. This was also confirmed by DFT calculations which suggested that the enzyme is responsible for providing $\sim 3.7$ $\mathrm{kcal} / \mathrm{mol}$ of activation for reduction from the ketone to the ketyl radical. To determine if this was a general phenomenon or limited to DBR enzymes, we turned back to the ketoreductase system previously developed and targeted $\alpha$ bromoamides. These represent substrates that were completely unreactive under our previous reaction conditions, presumably, because they do not form EDA complexes with NADPH. We hypothesized that binding the KRED should activate the amide for electron transfer. We were pleased to see that this strategy was effective. In the absence of photo- catalyst, no reaction was observed, however, upon addition of Eosin Y, the yield increased in $>60 \%$ with good to excellent levels of enantioselectivity (Scheme 10).

a.<smiles>CO[C@]1(C)CCc2ccccc2C1=O</smiles>

b.

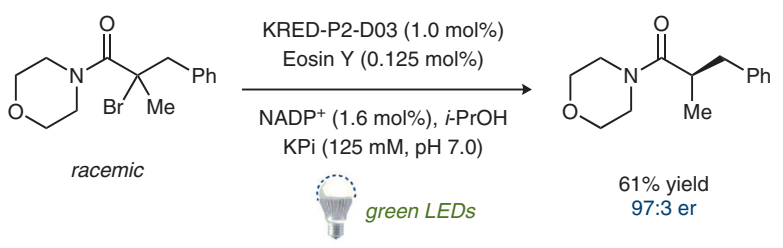

Scheme 10 Mechanistic experiments

\section{Conclusion}

In these studies, we have demonstrated how electron transfer can be used to reveal new enzyme functions. This represents a new strategy for achieving non-natural reactivity in enzyme active sites. As the catalysts employed are robust and commercially available, these reactions can be readily applied by synthetic chemists with little experience 
with synthetic biology. Studies are ongoing to expand this general type of reactivity to $\mathrm{C}-\mathrm{C}$ bond-forming reactions. As radical-mediated $\mathrm{C}-\mathrm{C}$ bond-forming reactions often resemble the ones catalyzed by organometallic intermediates, it is possible to imagine accessing this type of reactivity for common oxidoreductases rather than having to develop new artificial metalloenzymes. We are optimistic that this approach, in which reaction mechanisms from small molecule catalysis are merged with substrate promiscuous enzymes to access new biocatalytic functions, can be applied with other types of reactivity to provide new biocatalytic reactions to address challenges in chemical synthesis.

\section{Funding Information}

We thank Princeton University, the Searle Scholar Award, and the NIH (R01-GM127703) for support.

\section{Acknowledgment}

I would like to thank my group for their hard work in advancing this area of research. I would also like to thank Braddock Sandoval, Kyle Biegasiewicz, and Yuji Nakano for helpful comments on this manuscript.

\section{References}

(1) (a) Patel, R. N. Bioorg. Med. Chem. 2018, 26, 1252. (b) Martinez, C. A.; Hu, S.; Dumond, S.; Tao, J.; Kelleher, P.; Tully, L. Org. Process Res. Dev. 2008, 12, 392. (c) Savile, C. K.; Janey, J. M.; Mundorff, E. C.; Moore, J. C.; Tam, S.; Jarvis, W. R.; Colbeck, J. C.; Krebber, A.; Fleitz, F. J.; Brands, J.; Devine, P. N.; Huisman, G. W.; Hughes, G. J. Science 2010, 329, 305. (d) Greenberg, W. A.; Varvak, A.; Hanson, S. R.; Wong, K.; Huang, H.; Chen, P.; Burk, M. P. Proc. Natl. Acad. Sci. U.S.A. 2004, 101, 5788. (e) Liang, J.; Lalonde, J.; Borup, B.; Mitchell, V.; Mundorff, E.; Trinh, N.; Kochrekar, D. A.; Cherat, R. N.; Pai, G. G. Org. Process Res. Dev. 2010, 14, 193.

(2) (a) Devine, P. N.; Howard, R. M.; Kumar, R.; Thompson, M. P.; Truppo, M. D.; Turner, N. J. Nat. Rev. Chem. 2018, 2, 409. (b) Guazzaroni, M.-E.; Beloqui, A.; Golyshin, P. N.; Ferrer, M. World J. Microbiol. Biotechnol. 2009, 25, 945.

(3) (a) Hammer, S. C.; Knight, A. M.; Arnold, F. H. Curr. Opin. Green Sustain. Chem. 2017, 7, 23. (b) Renata, H.; Wang, Z. J.; Arnold, F. H. Angew. Chem. Int. Ed. 2015, 54, 3351. (c) Schwizer, F.; Okamoto, Y.; Heinisch, T.; Gu, Y.; Pellizzoni, M. M.; Lebrun, V.; Reuter, R.; Köhler, V.; Lewis, J. C.; Ward, T. R. Chem. Rev. 2018, 118, 142. (d) von Heijne, G. Annu. Rev. Biochem. 2018, 87, 101.

(4) (a) Bornscheuer, U. T.; Kazlauskas, R. J. Angew. Chem. Int. Ed. 2004, 43, 6032. (b) Miao, Y.; Rahimi, M.; Geertsema, E. M.; Poelarends, G. J. Curr. Opin. Chem. Biol. 2015, 25, 115. (c) Bornscheuer, U. T. Philos. Trans. A Math. Phys. Eng. Sci. 2018, 376, 20170063.

(5) (a) Hyster, T. K.; Ward, T. R. Angew. Chem. Int. Ed. 2016, 55, 7344. (b) Jeschek, M.; Panke, S.; Ward, T. R. Trends Biotechnol. 2018, 35,60 .

(6) Busto, E.; Gotor-Fernández, V.; Gotor, V. Chem. Soc. Rev. 2010, $39,4504$.

(7) (a) Sibi, M. P.; Manyem, S.; Zimmerman, J. Chem. Rev. 2003, 103, 3262. (b) Garrido-Castro, A. F.; Maestro, M. C.; Alemán, J. Tetrahedron Lett. 2018, 59, 1286. (c) Meggers, E. Chem. Commun. 2015, 51, 3290.

(8) Auclair, K.; Hu, Z.; Little, D. M.; Ortiz de Montellano, P. R.; Groves, J. T. J. Am. Chem. Soc. 2002, 124, 6020.

(9) Broderick, J. B.; Duffus, B. R.; Duschene, K. S.; Shepard, E. M. Chem. Rev. 2014, 114, 4229.

(10) Pollak, N.; Dölle, C.; Ziegler, M. Biochem. J. 2007, 402, 205.

(11) Zheng, C.; You, S.-L. Chem. Soc. Rev. 2012, 41, 2498.

(12) (a) Fukuzumi, S.; Hironaka, K.; Tanaka, T. J. Am. Chem. Soc. 1983, 105, 4722. (b) Fukuzumi, S.; Inada, S.; Suenobu, T. J. Am. Chem. Soc. 2003, 125, 4808.

(13) (a) Sibi, M. P.; Patil, K. Org. Lett. 2005, 7, 1453. (b) Nanni, D.; Curran, D. P. Tetrahedron: Asymmetry 1996, 7, 2417. (c) Murakata, M.; Tsutsui, H.; Hoshino, O. J. Chem. Soc., Chem. Commun. 1995, 481. (d) Tanner, D. D.; Kharrat, A. J. Am. Chem. Soc. 1988, 110, 2968. (e) Hague, M. B.; Roberts, B. P.; Tocher, D. A. J. Chem. Soc., Perkin Trans. 1 1998, 2881. (f) Nakano, Y.; Biegasiewicz, K. F.; Hyster, T. K. Curr. Opin. Chem. Biol. 2019, 49, 16.

(14) Emmanuel, M. A.; Greenberg, N. R.; Oblinsky, D. G.; Hyster, T. K. Nature 2016, 540, 414.

(15) Noey, E. L.; Tibrewal, N.; Jiménez-Osés, G.; Osuna, S.; Park, J.; Bond, C. M.; Cascio, D.; Liang, J.; Zhang, X.; Huisman, G. W.; Tang, Y.; Houk, K. N. Proc. Natl. Acad. Sci. U.S.A. 2015, 112, E7065.

(16) Man, H.; Kędziora, K.; Kulig, J.; Frank, A.; Lavandera, I.; GotorFernández, V.; Rother, D.; Hart, S.; Turkenburg, J. P.; Grogan, G. Top. Catal. 2014, 57, 356.

(17) Lima, C. G. S.; Lima, T. d. M.; Duarte, M.; Jurberg, I. D.; Paixão, M. W. ACS Catal. 2016, 6, 1389.

(18) Åhman, J.; Wolfe, J. P.; Troutman, M. V.; Palucki, M.; Buchwald, S. L. J. Am. Chem. Soc. 1998, 120, 1918.

(19) Toogood, H. S.; Scrutton, N. S. ACS Catal. 2018, 8, 3532.

(20) Mansoorabadi, S. O.; Thibodeaux, C. J.; Liu, H.-W. J. Org. Chem. 2007, 72, 6329.

(21) Sandoval, B. A.; Meichan, A. J.; Hyster, T. K. J. Am. Chem. Soc. 2017, 139, 11313

(22) (a) Tarantino, K. T.; Liu, P.; Knowles, R. R. J. Am. Chem. Soc. 2013, 135, 10022. (b) Lu, Z.; Shen, M.; Yoon, T. P. J. Am. Chem. Soc. 2011, 133, 1162.

(23) (a) Banerjee, A.; Falvey, D. E. J. Am. Chem. Soc. 1998, 120, 2965. (b) Banerjee, A.; Falvey, D. E. J. Am. Chem. Soc. 1997, 62, 6245

(24) Biegasiewicz, K. F.; Cooper, S. J.; Emmanuel, M. A.; Miller, D. C.; Hyster, T. K. Nat. Chem. 2018, 10, 770. 